\title{
La Relación del Hombre con su Primer(a) Hijo(a) Durante los Primeros Seis Meses de Vida: Experiencia Vincular del Padre
}

\section{Men's Relationship With Their First Child During the First Six Months of Life: Father's Bonding Experience}

\author{
Nicolás Suárez-Delucchi y Pablo Herrera \\ Pontificia Universidad Católica de Chile
}

\begin{abstract}
Se presentan los principales resultados de una investigación acerca de la experiencia de hombres en relación al vínculo con su primer(a) hijo(a) durante sus primeros 6 meses de vida. Se utilizó un diseño descriptivo-analítico relacional con metodología cualitativa, recolectando los datos mediante entrevistas individuales semiestructuradas a 14 padres, las que se analizaron a través de la grounded theory. Los resultados se agruparon en torno al fenómeno Experiencia de Conocerse Mutuamente, que implica el cuidado del padre al hijo(a), la confianza del hijo(a) hacia el padre y el afecto mutuo. Se discute la importancia de los factores que surgieron como intervinientes en la experiencia vincular gratificante: (a) cambios en la identidad, (b) relación con la madre y (c) tiempopresencia disponible.
\end{abstract}

Palabras clave: hombres, padres, experiencia, vínculo

The main findings of a study that explored the experience of bonding among men with their first child during the first six months of life are reported. This study was conducted using qualitative methodology within a descriptive-analytical design. Data was collected using semi-structured interviews with 14 fathers, which were analyzed using grounded theory. The results were organized around elements of the father's Experience of Getting to Know Each Other, including: the father's care of the child, the child's confidence in the father, and mutual affection. The relevance of the following emerging intervening factors for a rewarding bonding experience is discussed: (a) identity changes, (b) relationship with the mother, and (c) available time-presence.

Keywords: men, father, experience, bond

La mayor demanda social para la incorporación del hombre en tareas de crianza tradicionalmente femeninas ha motivado el interés emergente de diversos actores sociales, instituciones e investigadores sobre el fenómeno de la paternidad, incluyendo Latinoamérica y Chile (por ejemplo, en el Banco Mundial: Barker, 2003; en el Fondo de las Naciones Unidas para la Infancia: Aguayo, 2003; en la Facultad Latinoameri- cana de Ciencias Sociales: Olavarría, 2000). Este nuevo interés representa un cambio a nivel paradigmático, ya que anteriormente la preocupación se centraba primordialmente en el vínculo madre-hijo(a) y la figura del padre quedaba invisibilizada de los escenarios académicos y políticos.

Desde la psicología evolutiva es posible constatar el mismo fenómeno: distintas corrientes psicológicas, partiendo por el psi-

Nicolás Suárez-Delucchi y Pablo Herrera, Escuela de Psicología, Pontificia Universidad Católica de Chile, Santiago, Chile.

Este artículo reporta parte de los resultados de la tesis de magíster "Experiencia Vincular de los Hombres-Padres, en Relación con su Primer Hijo(a) Durante los Primeros 6 Meses de Vida de Este/a”, financiada en parte por el Proyecto FONDECYT N 1040760 . La tesis está disponible en la base de datos de la Pontificia Universidad Católica de Chile o directamente a través del autor principal.

Agradecemos la colaboración de Marcela Aracena, docente de la Escuela de Psicología de la Pontificia Universidad Católica de Chile; Esteban Gómez, Gonzalo Gallardo y Luis Felipe Muñoz, psicólogos de la Pontificia Universidad Católica de Chile; y Sylvia Palma, matrona del Hospital Sótero del Río.

La correspondencia relativa a este artículo debe ser dirigida a Nicolás Suárez-Delucchi, Escuela de Psicología, Pontificia Universidad Católica de Chile, Avda. Vicuña Mackenna 4860, Santiago, Chile. E-mail: nsuarez@uc.cl, nicolasuarez@gmail.com 
coanálisis, han dado énfasis a las relaciones tempranas que tiene el bebé y su entorno familiar más cercano para el desarrollo adecuado en su vida adulta (Bowlby, 1998). Aunque Bowlby (1998) plantea la idea de que el cuidador primario no necesariamente es la madre, esta ha sido tradicionalmente la figura de apego central en la cultura occidental. La mayoría de las investigaciones se centra en cómo se da esta relación y qué tipo de vínculo se construye entre la madre y su hijo(a), quedando el padre muchas veces excluido de las teorías e investigaciones respecto del vínculo (Maldonado-Durán, 2005; Pruett, 1998).

Desde hace algún tiempo en países como Francia, Reino Unido, Estados Unidos y Canadá se ha abordado la relación del padre con el bebé, habiendo, sin embargo, escasas investigaciones tanto recientes como pasadas que profundicen en esta relación (Maldonado-Durán, 2005; Pruett, 1998). Pruett (1998) plantea en sus recomendaciones para la investigación y la práctica la necesidad crítica de profundizar en el desarrollo de un paradigma comprensivo sobre la paternidad. Dentro de este contexto, la investigación que se presenta busca aportar al desarrollo de dicho paradigma, enfocándose en la manera en que los hombres significan la experiencia vincular con su primer hijo durante sus primeros seis meses de vida. Para ello se utiliza metodología cualitativa, con entrevistas semiestructuradas a 14 padres primerizos, analizadas según los principios de la grounded theory (Strauss \& Corbin, 2002).

\section{Antecedentes Teóricos y Empíricos}

A continuación se presentan tres corrientes de investigación acerca de la paternidad desde las cuales se construyeron los marcadores teóricos y empíricos que guiaron el presente estudio. En primer lugar, se fundamenta desde la teoría del apego la importancia del vínculo temprano entre el padre y sus hijos(as $)^{1}$, presentando las

\footnotetext{
1 Los autores asumen la importancia de la distinción lingüística de género, referida a hijo varón o hija mujer; sin embargo, para facilitar la lectura del texto, en adelante esta será obviada, optando por el término genérico hijo.
}

diferencias observadas entre el padre y la madre. Posteriormente, se consideran los aportes latinoamericanos que derivan de los estudios de significados y representaciones sociales asociados al concepto de paternidad y cómo estos influyen en la identidad masculina. Luego, se ahonda en los conceptos teóricos e investigaciones sobre la experiencia de los hombres en el período de transición a la paternidad, realizadas principalmente durante el período del embarazo, que se relacionan de manera directa con esta investigación.

\section{Importancia del Apego Entre el Padre y sus Hijos: Diferencias con la Madre al Observar Interacciones Tempranas}

Dentro de los ejes teóricos que exploran el vínculo paterno-filial, la teoría del apego cobra relevancia, ya que en esta se destaca la importancia de los vínculos afectivos en el desarrollo de nuestra especie (Bowlby, 1998); de esta forma, plantea que existe en el hombre una necesidad constitucional de tocar y aferrarse a otro ser humano de manera de sentirse protegido y cuidado. Así, el apego es considerado como una pulsión muto propio, no subordinada a las gratificaciones libidinales de tipo oral, como se propone en la teoría freudiana (Marrone, 2001).

Según Stern (1978), los niños cuentan con un repertorio de conductas innatas que promueven acciones coordinadas, tales como la mirada, los movimientos de cabeza y las expresiones faciales. Federico (2003) destaca la importancia de la audición, como primer sentido que permite, desde el embarazo, el contacto del bebé con el mundo que lo rodea y con las figuras de apego.

Para poder gratificar la necesidad de apego es importante que los cuidadores puedan dar respuestas sensibles, que consisten en notar las señales del bebé y dar una adecuada interpretación y respuesta a ellas. Otra función importante consistiría en ofrecerse como una base segura en la que el niño puede recurrir para satisfacer sus necesidades de apego y, al mismo tiempo, permitir el desarrollo de conductas exploratorias (Marrone, 2001).

Uno de los primeros autores en estudiar a los padres como figuras de apego fue Michael 
Lamb (1997). Además de concluir que ellos también podían ser figuras adecuadas de apego, observó que las interacciones con sus hijos presentaban cierta especificidad, siendo estas más estimulantes físicamente e impredecibles, en comparación con las de las madres (Clarke-Stewart, 1978; Lamb, 1997). Estos hallazgos se han encontrado consistentemente en la cultura occidental, no siendo necesariamente así en otras culturas (Lamb, 1997).

En esta misma línea, Gibbons et al. (2001) encontraron que los niños eran más claros en sus señales y respondían más a los acercamientos de los padres que a los de sus madres, aunque las madres eran más sensibles a las señales de sus hijos. Kazura (2000) reporta que, aunque los padres no estaban tan involucrados como las madres en el cuidado de los niños, estos desarrollaban mejores interacciones lúdicas con sus hijos. Respecto de los determinantes en el involucramiento responsable del padre con su bebé, Volling y Belsky (1991) señalan que hay múltiples factores determinantes, entre los que están la autoestima y empatía paterna, el temperamento del hijo, la posibilidad de complementar trabajo y familia, junto con la relación marital. Este último factor influye en mayor medida en el involucramiento de los padres que de las madres con sus bebés (Belsky, Yungblade, Robin \& Volling, 1991).

Las diferencias encontradas entre el padre y la madre a partir de los estudios de observación de interacciones plantean la interrogante sobre la experiencia subjetiva de los padres derivada de la construcción de significados en torno al vínculo, que es lo que se aborda en este artículo.

\section{Significados y Representaciones Sociales Asociados a la Paternidad}

Investigaciones sobre la masculinidad y la paternidad realizadas en diversos países de Latinoamérica, utilizando metodología cualitativa, estudian la paternidad en términos psicosociales, centradas en sus significados y relación con el sistema social sexo-género (Alatorre \& Luna, 2000; Cruzat, 2003; Escobar \& Muñoz, 1995; Fachel, 2000; Fuller, 2000a; Gallardo, Gómez, Muñoz \&
Suárez, 2006; Mora, Otárola \& RecagnoPuente, 2005; Olavarría, 2000; Viveros, 2000). Estas investigaciones integran la diversidad de las experiencias de los padres, mostrando que en la construcción de la identidad masculina la paternidad emerge como un eje central (Fuller, 2000b). Más allá de las diferencias en las prácticas concretas, el padre se concibe como:

... aquel que protege, provee, forma y educa (...) [Asimismo, la paternidad] se vive como el momento en que se cierra la etapa juvenil, significa un reordenamiento de la vida del varón y su inserción a un nuevo período en el que obtiene pleno reconocimiento social, es el punto en el que un varón se convierte en un adulto. (Fuller, 2000b, p. 28)

En la misma línea "aparece un nuevo mandato moral que se resume en dos grandes demandas: diálogo horizontal entre padres e hijos/hijas y mayor participación del padre en la crianza de los hijos" (Fuller, 2000b, p. 28).

En relación a este nuevo modelo ideal de paternidad, un número creciente de hombres expresa su incompetencia para desempeñarse de acuerdo a las exigencias y expectativas actuales sobre su rol como padre, atribuyéndolo a las largas jornadas laborales (Olavarría, 2001).

Entre los hallazgos realizados en investigaciones con poblaciones chilenas y latinoamericanas que han explorado específicamente la relación padre-hijo y a las personas que podrían influir en este vínculo, es posible destacar las siguientes conclusiones: (a) la paternidad se consagra al tener un hijo varón, ya que permite que el apellido de la familia se trasmita y sobreviva en el tiempo, aunque el vínculo amoroso sea con la madre (Fuller 2000a; Olavarría, 2000); (b) la hija mujer representa el vínculo familiar, la solidaridad y estaría más ligada a la madre y (c) la relación con las hijas se percibe con mayor profundidad emocional que la con los varones (Fuller, 2000a).

Respecto del impacto sobre la madre del hijo, las investigaciones señalan que el hecho de convertirse en padre rearticula la relación de pareja, convirtiéndola en una relación de familia, señalando, además, 
que el vínculo con la pareja condicionaría la forma como se vivencia la paternidad (Alatorre \& Luna, 2000; Escobar \& Muñoz, 1995; Fuller, 2000a; Gallardo et al., 2006; Mora et al., 2005).

En relación a las experiencias pasadas, tanto el padre como la madre son referentes para los hombres en su relación con sus hijos, a partir de las propias vivencias y enseñanzas (Cruzat \& Aracena, 2006; Fuller, 2000a; Gallardo et al., 2006; Olavarría, 2000).

\section{La Experiencia de los Hombres al Convertirse en Padres}

Los procesos psicológicos acontecidos tanto en el hombre como en la mujer durante el embarazo y los primeros meses de vida de su hijo han sido descritos dentro del proceso de transición a la parentalidad (o parentificación), afirmando Stoleru (1995) que “...es el reconocimiento del niño como tal lo que induce el desarrollo del sentimiento de ser padre: reconocimiento de la dependencia e imposibilidad del niño de satisfacer sus propias necesidades" (p. 94). Esta transición no solo concierne a la mujer, sino que incluye al padre, ya que, como plantea Federico (2003), el embarazo no se da solo en la esfera física sino, también, en las esferas mental, emocional y espiritual.

Una conceptualización complementaria al proceso de parentificación la ofrecen Cupa y Riazuelo-Deschamps (2001), basándose en el concepto psicoanalítico de constelación maternal (Stern, 1997). Ellos postulan que, en torno al nacimiento de su hijo, los padres pueden desarrollar una profunda reorganización subjetiva, denominada constelación paternal. Dicha constelación estaría constituida por preocupaciones paternales primarias (asegurar el crecimiento de la vida del bebé, su desarrollo psíquico y la capacidad de comprometerse con el bebé), además de una red de apoyo con la madre del bebé o ciertas figuras masculinas o femeninas, destacando la importancia de las interacciones con el bebé antes del nacimiento, así como las nuevas identificaciones paternas. A diferencia de lo que plantea Stern (1997), quien propone que en la constelación maternal el complejo de Edipo no juega un rol estructurante, la problemática edípica en estas reorganizaciones es un componente central de la constelación paternal: el hijo es percibido por el padre como un rival o competidor por el afecto de la cónyuge (Cupa \& Riazuelo-Deschamps, 2001).

Respecto a los factores que inciden en el desarrollo de la constelación paternal, en un estudio con 12 padres a los siete meses de embarazo de su pareja, Cupa y RiazueloDeschamps (2001) hallaron que la percepción de los movimientos del hijo es clave para sentirlo real y presente. Los padres mostraron una preferencia marcada por los niños varones, lo que relacionaron con que llevará su apellido y será su sucesor en la transmisión de este. Otro estudio que profundiza en la experiencia de padres durante el embarazo y primeros meses de su hijo concluye que, al participar en la confirmación del embarazo, los padres acceden a la dimensión física de este, lo que les ayuda a sentirse más comprometidos con su hijo por nacer (Draper, 2002).

Herzog y Lebovici (1995) enfatizan la relación de apoyo mutuo que debe existir en la pareja, señalando que uno de los procesos más importantes de la transición a la paternidad tiene que ver con la redefinición de la identidad de hombre y esposo hacia padre. Entre los procesos psicológicos que esta tarea implica, además de crear un lazo con el bebé, el padre también debe lograr la convicción interna de que puede cuidar de él. Los autores señalan que el padre puede ayudar a la madre en el proceso de transición a la parentalidad, pero él también tiene necesidad de sostén para paternalizarse. Además de necesitar del apoyo afectivo de la madre para volverse un padre interesado, el proceso de paternalización estaría facilitado por la participación del padre en el deseo de tener un hijo, por la preparación para el parto y por su presencia en la sala de parto.

Además de los estudios sobre el vínculo padre-hijo, hay otros que profundizan en la experiencia de los padres durante el primer embarazo de su pareja. Barclay, Donovan y Genovese (1996) señalan que la mayoría de los padres se sentían confundidos respecto a lo que se espera de ellos durante el embarazo. Estaban desconcertados por los cam- 
bios en la relación con su pareja y sentían que sus roles respecto del bebé y de otras personas eran poco claros. La percepción de sí mismos (self) se sentía amenazada y percibían negativamente los cambios experimentados, en especial el distanciamiento de su pareja. Los hombres sentían, además, que los servicios clínicos y educacionales los dejaban de lado. Este hallazgo de la despreocupación percibida coincide con otra investigación cualitativa, realizada por Bradley, Mackenzie y Boath (2004). Los autores reportan que en las clases de preparación para el nacimiento los padres se sentían presionados a descuidar su propio soporte personal a favor de su compañera. Esto es similar a lo reportado por padres adolescentes chilenos, quienes expresan sentirse descuidados por los servicios sociales y de salud, en pos de la madre del hijo (Cruzat, 2003).

Otro hallazgo es que algunos padres sienten el nacimiento de su hijo como un cambio radical en su vida, estresante y en general con connotación negativa, mientras que los que logran incorporar a su hijo en sus planes tienden a ver esta nueva etapa de manera más optimista (Bradley et al., 2004), lo que concuerda, a su vez, con los resultados encontrados por Escobar y Muñoz (1995) en Chile.

\section{El Vínculo Temprano a Través del Significado de la Experiencia Paterna en la Relación con su Hijo Después de Nacido}

Las investigaciones revisadas profundizan en las interacciones tempranas desde la perspectiva del observador (e.g., Kazura, 2000; Lamb, 1997; Volling \& Belsky, 1991), en los significados y experiencias de padres acerca de la paternidad e identidad de género (e.g., Alatorre \& Luna, 2000; Cruzat \& Aracena, 2006; Escobar \& Muñoz, 1995; Fuller, 2000a; Olavarría, 2000) o en su experiencia del vínculo con su hijo durante el período de embarazo (e.g., Barclay et al., 1996; Cupa \& Riazuelo-Deschamps, 2001; Draper, 2002). A diferencia de estas, la presente investigación buscó ahondar en los procesos psicológicos y emocionales asociados a la experiencia vincular desde la perspectiva de los propios padres, no solo en la etapa del embarazo, sino abarcando los primeros meses de vida de la etapa de transición a la paternidad (Stoleru, 1995), un área que se ha estudiado insuficientemente (Maldonado-Durán, 2005).

Específicamente, la investigación aquí presentada tuvo como objetivo describir y analizar la forma en que los hombres significan su experiencia vincular con su primer hijo durante los primeros seis meses de su vida.

\section{Método}

\section{Diseño}

Para realizar la investigación se utilizó un diseño descriptivo-analítico relacional y se empleó metodología cualitativa. De acuerdo a Flick (2004), la manera como los individuos revisten de significado sus experiencias constituye el punto de partida para la investigación cualitativa. Es por ello que esta metodología resultó pertinente para esta investigación, en la medida en que permitió acceder a los significados subjetivos de los hombres que son padres por primera vez, con el fin de comprender a través de ellos la experiencia vincular con su hijo.

\section{Participantes}

El muestreo utilizado fue no probabilístico, de carácter intencional en base a criterios de inclusión y de heterogeneidad (Patton, 1990). Los criterios de inclusión fueron elegidos en forma coherente con el objeto de estudio, intentando que la muestra fuera lo más homogénea posible, considerándose además criterios de conveniencia asociados a la posibilidad de acceso a la muestra. En términos específicos se incluyeron (a) hombres adultos chilenos que eran padres por primera vez; (b) cuyo hijo tenía entre uno y seis meses de edad, de modo de acceder a padres que estuvieran en el período de transición a la paternidad posterior al embarazo; (c) que vivían con su hijo y con la madre de este, independientemente si eran casados o no, ya que se profundizó en la experiencia de padres que tuvieran un contacto estable y permanente con sus hijos; (d) residentes en las comunas de Puente Alto o 
La Florida y (e) con enseñanza media completa (algunos de ellos con estudios profesionales en áreas técnico-profesionales), de manera que el nivel socio-cultural fuera lo más homogéneo posible.

La muestra se determinó en base al criterio de saturación teórica de las categorías conceptuales centrales y de las relaciones entre ellas. La saturación teórica puede definirse como el momento en que los datos recogidos ya no logran aportar nuevas características a las categorías conceptuales descubiertas (Strauss \& Corbin, 2002). De este modo, la muestra estuvo constituida por 14 padres, 7 con hijos hombres y 7 con hijas mujeres. El detalle de la muestra se puede observar en la Tabla 1.

\section{Instrumento}

El instrumento utilizado fue una entrevista individual semiestructurada que permitió obtener información de los padres acerca de los siguientes tópicos: establecimiento del vínculo con su hijo o hija, identidad de padre a partir del vínculo con su hijo y vínculos significativos actuales y pasados que han influido en la relación con su hijo.

Tabla 1

Caracterización de la Muestra

\begin{tabular}{|c|c|c|c|c|c|c|}
\hline $\begin{array}{l}\text { Edad } \\
\text { padre }\end{array}$ & $\begin{array}{l}\text { Edad } \\
\text { hijo(a) }\end{array}$ & $\begin{array}{l}\text { Sexo } \\
\text { hijo(a) }\end{array}$ & $\begin{array}{l}\text { Estado } \\
\text { civil }\end{array}$ & $\begin{array}{c}\text { Nivel } \\
\text { educacional }\end{array}$ & Ocupación & Comuna \\
\hline 26 & $5 \mathrm{~m} \mathrm{20d}$ & Masculino & Casado & $\begin{array}{l}\text { Enseñanza } \\
\text { media }\end{array}$ & $\begin{array}{c}\text { Cesante } \\
\text { (manipulador } \\
\text { alimentos) }\end{array}$ & Puente Alto \\
\hline 23 & $4 \mathrm{~m} 11 \mathrm{~d}$ & Masculino & Convive & $\begin{array}{l}\text { Enseñanza } \\
\text { media }\end{array}$ & Obrero Soldador & Puente Alto \\
\hline 29 & $4 \mathrm{~m} 15 \mathrm{~d}$ & Femenino & Casado & Profesional & Ingeniero Ejecución & Puente Alto \\
\hline 24 & $4 \mathrm{~m} 13 \mathrm{~d}$ & Femenino & Casado & $\begin{array}{l}\text { Enseñanza } \\
\text { media }\end{array}$ & Vendedor & Puente Alto \\
\hline 33 & $5 \mathrm{~m} 20 \mathrm{~d}$ & Femenino & Casado & Profesional & Ingeniero Ejecución & La Florida \\
\hline 25 & $5 \mathrm{~m} 4 \mathrm{~d}$ & Masculino & Convive & Profesional & $\begin{array}{l}\text { Administrador } \\
\text { Empresa Familiar }\end{array}$ & Puente Alto \\
\hline 23 & $6 \mathrm{~m} 1 \mathrm{~d}$ & Femenino & Convive & $\begin{array}{l}\text { Enseñanza } \\
\text { media }\end{array}$ & $\begin{array}{l}\text { Obrero Estaciones } \\
\text { Metálicas }\end{array}$ & Puente Alto \\
\hline 22 & $6 \mathrm{~m} \mathrm{14d}$ & Masculino & Convive & $\begin{array}{l}\text { Enseñanza } \\
\text { media }\end{array}$ & $\begin{array}{l}\text { Operario plaza } \\
\text { pública }\end{array}$ & Puente Alto \\
\hline 24 & $6 \mathrm{~m} 10 \mathrm{~d}$ & Femenino & Convive & $\begin{array}{c}\text { Técnico } \\
\text { profesional }\end{array}$ & $\begin{array}{l}\text { Técnicos Mecánico } \\
\text { Industrial }\end{array}$ & Puente Alto \\
\hline 24 & $1 \mathrm{~m} \mathrm{3d}$ & Masculino & Convive & Profesional & Contador Auditor & La Florida \\
\hline 21 & $1 \mathrm{~m} 5 \mathrm{~d}$ & Femenino & Casado & $\begin{array}{c}\text { Técnico } \\
\text { profesional }\end{array}$ & Obrero construcción & Puente Alto \\
\hline 22 & $1 \mathrm{~m} 8 \mathrm{~d}$ & Masculino & Convive & $\begin{array}{l}\text { Enseñanza } \\
\text { media }\end{array}$ & Vendedor & Puente Alto \\
\hline 26 & $1 \mathrm{~m} 7 \mathrm{~d}$ & Femenino & Casado & $\begin{array}{c}\text { Técnico } \\
\text { profesional }\end{array}$ & $\begin{array}{l}\text { Administrador } \\
\text { de Empresa } \\
\text { informática }\end{array}$ & Puente Alto \\
\hline 22 & $1 \mathrm{~m} 5 \mathrm{~d}$ & Masculino & Convive & $\begin{array}{c}\text { Enseñanza } \\
\text { media }\end{array}$ & Pintor automóviles & Puente Alto \\
\hline
\end{tabular}




\section{Procedimiento}

Las entrevistas fueron realizadas por el investigador principal en una sala habilitada para tales fines, fuera del lugar de residencia o trabajo del participante. Las entrevistas tuvieron una duración promedio de 75 minutos.

El estudio contempló las siguientes consideraciones éticas: (a) consentimiento informado escrito, informando a los padres sobre los objetivos de la investigación; (b) resguardo de la información, asegurando la confidencialidad a cada participante, y (c) estrategias de derivación en el caso de que alguno de los padres lo requiriera. Los participantes no requirieron de un acompañamiento adicional finalizado el estudio.

\section{Análisis de Datos}

El análisis se realizó según los procedimientos estipulados en la grounded theory (Strauss \& Corbin, 2002). En primer lugar, se realizó una codificación abierta, correspondiente al examen y fragmentación de las entrevistas transcritas para clasificar el material en conceptos y categorías que agrupan toda la información recolectada, la que, al ser sintetizada, dio lugar a los resultados descriptivos. Para comprender estos resultados y explicar el fenómeno de estudio se realizó una codificación axial, con la cual dichos conceptos se re-agruparon en modelos conceptuales más complejos, usando un paradigma de análisis que incluye el fenómeno central, su contexto general de aparición, las condiciones causales intervinientes, las estrategias de acción o interacción y, finalmente, sus consecuencias. La codificación axial permitió tener un primer nivel de resultados relacionales, los cuales posteriormente se sintetizaron usando el procedimiento de codificación selectiva, generando un modelo explicativo en torno al fenómeno central del estudio. Es este el que se presenta en este artículo.

La triangulación de la codificación realizada por los investigadores (validación intersubjetiva) fue la estrategia que permitió asegurar la calidad y rigurosidad de los resultados al recolectar los datos y analizarlos, en pos de la consistencia interna y ri- gor científico de la investigación (Pedersen, 1992).

\section{Resultados}

A continuación se presentan los resultados obtenidos, diferenciándolos según nivel de análisis y dando prioridad a los resultados finales del estudio. Un primer nivel de análisis (codificación abierta) permitió describir cuatro categorías relevantes: (a) caracterización del vínculo con el hijo; (b) influencia del vínculo en la identidad de padre; (c) influencia de personas significativas actuales y (d) influencia de experiencias significativas pasadas, tanto con otros como consigo mismo. Para dar lugar a resultados relacionales, estos resultados descriptivos se analizaron por medio del proceso de codificación axial, generando dos modelos comprensivos emergentes del discurso de los padres asociados al objetivo del estudio: el primero, referido a la identidad paterna, es el sentimiento de "padre" como responsable del bienestar total del hijo; el segundo, referido a las personas influyentes en el vínculo, se denomina madre del hijo como influencia fundamental en la experiencia vincular con este.

Estos primeros resultados relacionales se sintetizaron mediante el procedimiento de codificación selectiva en torno al fenómeno central del estudio: la experiencia de conocerse padre e hijo mutuamente (ver la Figura 1). El modelo central ilustra que los padres significan su vínculo con su primogénito como una experiencia de conocerse mutuamente e incluye la descripción de cómo se da este proceso (contacto sensorial directo), las condiciones que permiten este conocimiento mutuo (capacidad/incapacidad paterna), los principales factores intervinientes (presencia disponible, cambios en la identidad y relación con la madre) y las consecuencias, tanto si se desarrolla de manera gratificante como no gratificante.

La experiencia de conocerse padre $e$ hijo mutuamente incluye tres procesos: el cuidado del padre al hijo, la confianza del hijo hacia el padre y el afecto mutuo. Este conocimiento mutuo se da a través del contacto sensorial directo con el hijo. Dentro de las principales formas de contacto sensorial 


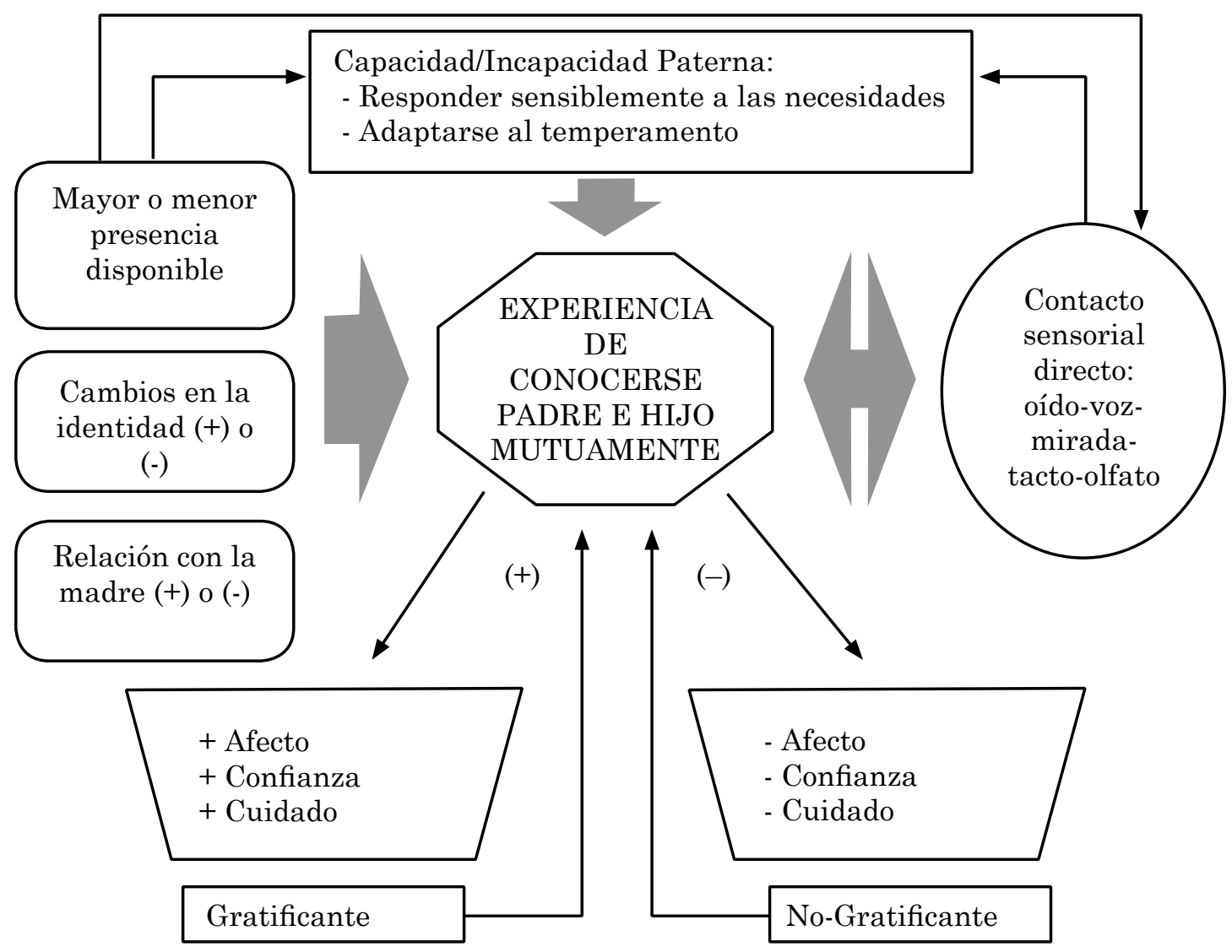

Figura 1. Experiencia de conocerse padre e hijo mutuamente.

que sostienen el conocimiento mutuo están percibir / ser percibido por el hijo, las manifestaciones físicas mutuas de cariño y la comunicación auditiva. La participación del hijo en este proceso va aumentando desde el embarazo (mínima) hasta que el hijo nace y luego a medida que va creciendo (mayor). Si este contacto sensorial directo ocurre en base a un conocimiento mutuo, surgen sensaciones gratificantes para el padre que permiten el desarrollo del afecto mutuo.

....yo lo cambié, y mientras lo estoy cambiando, él empieza como a balbucear: "agu-ag", entonces, mientras yo le hablo, yo le digo: "hijo, yo te quiero mucho, estoy feliz de que estés aquí, soy feliz cuando llego a la casa y cuando en los controles me dicen que eres inteligente", entonces, yo le converso, le converso, le digo que lo queremos mucho que "tu mamá te quiere mucho, yo te quiero mucho" y él me mira, chupa su mano, se chupa un dedo, me mira y empieza: "ua ua"; entonces me da la sensación de que yo estoy conversando con un amigo, y eso me, en el momento, claro, en el momento me dan ganas de llorar de alegría y de felicidad, $y$ en ese momento, especificamente sería, porque como hablaba, con la detención que me mira, la atención que me presta... (II, 32) ${ }^{2}$

La experiencia de conocerse mutuamente puede darse de manera positiva, dando lugar a una experiencia gratificante, o de manera negativa, lo que se traduce en una experiencia no gratificante. ¿Qué es lo que determina que la experiencia sea gratificante o no gratificante? Se pueden distinguir ciertas condiciones y algunos factores que intervienen. Dentro de las condiciones

2 Se indica en número romano al padre entrevistado (desde I a XIV) y en número arábico el párrafo de la entrevista en el que aparece la cita. 
que permiten a los hombres este conocimiento mutuo está la progresiva capacidad/ incapacidad paterna para responder sensiblemente a las necesidades y adaptarse al temperamento del hijo. Así, el padre, en el cual se expresan dichas capacidades, podrá acercarse al hijo de mejor manera, permitiéndole ser reconocido por el hijo como un agente de cuidado y, de esta manera, obtener también la confianza del hijo:

De primera no teníamos idea, ahora ya se sabe, por ejemplo, si el niño alega y hace algún gesto (...) tiene hambre o tiene un chancho, entonces, si tú le das, le pones el dedito en la boca y él chupa es porque tiene hambre, entonces, tú le das la mamadera y ningún problema (...) En el caso de que el niño alegue, alegue $y$, no sé, y no es por eso, es porque hay que cambiarlo, está pasao, tú lo vai tocando, si está mojadito o no, y a veces él, tan solo de tú acercarte y hablarle, ya se queda quieto, eso ya es cuando se siente solo. Alega y, si tú le hablas, te mira y se queda quieto, se queda tranquilo. (X, 22)

Los factores que intervienen en la experiencia de conocerse son, en primer lugar, la relación con la madre, ya que esta facilita los procesos de conocimiento mutuo (entrega claves, pone al día al padre), además de incentivar el contacto sensorial entre el padre y el hijo, tanto con su presencia como con el incentivo de la relación dual. Si hay una relación disfuncional con la madre, estos procesos facilitadores se verían afectados, tal como lo señala un entrevistado:

Por ejemplo, algunas veces cuando no lo puedo calmar, no lo puedo calmar (...) me dice: "ipero por qué siempre llora cuando está en tus brazos?" Chuta, no siempre llora, no siempre llora cuando está en mis brazos. Entonces, yo siento que no ve nada de lo que yo hago, ahi dificulta. (XII, 174)

Otro factor interviniente es cómo los padres significan los cambios en la identidad durante la espera y el nacimiento del hijo. Para los padres entrevistados la llegada de su primogénito/a implica el sentirse padre, responsable por una entrega total a su hijo. Sin embargo, esto se puede vivenciar de dos maneras distintas: como una oportunidad de trascendencia o como una restricción de la libertad personal. Si el padre tenía en su proyecto vital el anhelo de tener un hijo, los cambios en la identidad tenderán a vivenciarse como positivos. En este caso, ser padre se experimentará positivamente, asociado a una experiencia de entrega total y visión trascendente de la vida, y aumentará la disposición y capacidad del padre para conocer a su hijo así como las estrategias de contacto sensorial mutuo. Además, el padre sentirá cambios positivos en la identidad, como mayor madurez, más energía y mayor autoestima. Si los cambios son vividos de manera negativa y predomina una sensación de pérdida de libertad, habrá un menor disfrute e interés por el contacto sensorial y el conocimiento del hijo se tornará más arduo.

Un tercer factor interviniente fundamental es la presencia disponible con el hijo. Mientras más presente esté el padre, con mayor facilidad podrá contactarse sensorialmente con el hijo, así como este podrá contactarse con su padre, aumentando el afecto mutuo. También podrá conocer mejor a su hijo y permitir que el hijo lo conozca a partir del desarrollo de su capacidad. Por esto la actividad laboral aparece como uno de los principales obstáculos para contactarse con el hijo:

Es que porque, porque de repente la pienso, trabajo y no puedo pasar con él. Es que me gustaría a mí como padre, me gustaría pasar todo el tiempo con él, pero no puedo, porque tengo que trabajar pa tenerle las cosas a él. Me gustaría, esa es la rabia, de repente, por eso el día libre lo aprovecho harto con él... (VIII, 39)

En consecuencia, a partir del análisis de participantes que reportaron experiencias gratificantes y no gratificantes, se puede afirmar que si estos tres factores intervinientes se dan en forma positiva, se desarrollará un buen conocimiento mutuo, lo que significa que habrá una relación caracterizada por el cuidado del padre hacia el hijo y, por lo tanto, se desarrollará la confianza del hijo hacia el padre, lo que derivará, a su vez, en un desarrollo potencial del afecto mutuo y se tornará gratificante para el padre. De esta forma, lo gratificante de la 
relación retroalimentará los deseos de contacto sensorial directo y, por ende, el padre mejorará su capacidad de adaptarse a su hijo. Si, en cambio, estos tres factores intervinientes se dan de forma negativa (padre poco disponible, cambios en la identidad vivenciados como restricción de la libertad personal y mala relación con la madre del hijo), el vínculo se verá afectado, por lo que el padre se sentirá menos motivado para conocer a su hijo. Luego, el contacto sensorial en sí mismo será menos gratificante, lo que podría influir en un menor cuidado del padre, afectando el desarrollo de la confianza del hijo y el afecto mutuo, pudiendo el padre, en algunos casos, vivir la relación como extraña:

\begin{abstract}
... la relación que tengo con él ahora ha sido un poco, no sé, ha sido extraña, ha sido muy extraña porque quizás fue el cambio muy fuerte al momento de que, no sé, que cambió mi vida un 100\%, 360 grados, porque algunas veces siento que no entiendo por qué está más con ella, yo sé por qué, pero no lo entiendo, que yo no puedo estar más con él, así sentirlo más, entonces, algunas veces entro en conflicto $y$, no sé, unas veces me da pena no poder quererlo tanto, tanto como ella. (XII, 1)
\end{abstract}

\section{Discusión}

En síntesis, los padres del estudio significan su experiencia vincular con su primer hijo como una experiencia de conocimiento mutuo, que incluye el cuidado del padre al hijo, la confianza del hijo en el padre y el afecto mutuo. Dicha experiencia se da a través del contacto sensorial directo con el hijo y es condicionada por la progresiva capacidad paterna de responder sensiblemente a las necesidades y adaptarse al temperamento del hijo.

Si los factores intervinientes, como la relación con la madre, la significación de los cambios en la identidad y la presenciatiempo disponible con el hijo, se presentan en forma positiva, se desarrollará un buen conocimiento mutuo. Por lo tanto, habrá una relación caracterizada por el cuidado del padre hacia el hijo y se desarrollará la confianza del hijo hacia el padre, lo que de- rivará, a su vez, en un desarrollo potencial del afecto mutuo y se tornará una experiencia gratificante para el padre.

Considerando la importancia dada a las relaciones tempranas desde la teoría del apego (Bowlby, 1998; Marrone, 2001) y coincidiendo con los postulados de Lamb (1997) acerca de que los hombres pueden ser figuras de apego adecuadas, es posible concluir que los padres del presente estudio se consideran a sí mismos figuras de apego, ya que en su experiencia de conocimiento mutuo es fundamental que el hijo sienta confianza y lo distinga de otras personas, estableciendo una relación de exclusividad, destacando la posibilidad de desarrollar la capacidad de satisfacer las necesidades afectivas del hijo y dar respuestas sensibles.

Aunque los padres no consideran que haya mayores diferencias respecto de la madre en el vínculo con su hijo, sí plantean que se sienten menos competentes que ella para sus cuidados y que juegan con su hijo o lo estimulan más que ella. Sin embargo, los padres no coinciden con la idea de que habría un modo específico de relación con sus bebés, propio de los hombres y diferente al de las mujeres. Ello difiere de lo señalado por los teóricos de interacciones precoces, quienes atribuyen diferencias de género (Clarke-Stewart, 1978; Herzog \& Lebovici, 1995; Lamb, 1997). La explicación que los mismos padres dan es que estas diferencias se deben más bien al menor tiempo-presencia que comparten con su hijo, a diferencia de la madre, y que en ocasiones se sienten más incompetentes que ella para cuidarlos, porque los conocen menos. Lo anterior sería coherente con las conclusiones de Bowlby (1998) sobre que el cuidador principal es quien está más disponible y atento al hijo; no necesariamente la madre.

A partir de estos resultados, es importante destacar que para los padres el trabajo es uno de los mayores obstáculos para vincularse con su hijo, ya que impide una mayor presencia disponible, lo que coincide con lo planteado por Volling y Belsky (1991) y Olavarría (2001).

La experiencia de los padres durante los primeros meses de vida de su primer hijo es concordante con el proceso descrito como transición a la parentalidad, ya que, de 
acuerdo a Stoleru (1995), el reconocimiento de la dependencia del hijo y la imposibilidad de satisfacer las propias necesidades es lo que caracteriza a este sentimiento. Herzog y Lebovici (1995), por su parte, señalan que el proceso de paternalización estaría facilitado por la participación del padre durante el embarazo, por la preparación para el parto y por su presencia en la sala de parto, lo que coincide con los hallazgos de la presente investigación en relación a las etapas del establecimiento del vínculo. Los resultados de la presente investigación son consistentes con los de Cupa y Riazuelo-Deschamps (2001) y Draper (2002) respecto a la importancia para la experiencia vincular de las interacciones padre-bebé durante el embarazo y su consecuente anticipación de la relación futura. Respecto al período del embarazo, los hallazgos concuerdan con los planteamientos de Federico (2001), ya que los padres reconocen la importancia de hablar al hijo durante el embarazo para el establecimiento del conocimiento mutuo.

En relación al postulado de la constelación paternal (Cupa \& Riazuelo-Deschamps, 2001), la experiencia de los padres que participaron en esta investigación es consistente con dos de los aspectos de dicha teoría. Por una parte, asegurar que el hijo se mantenga vivo, además de preocuparse por su desarrollo psíquico, y, por otra, en el reordenamiento de la identidad del hombre en torno al nacimiento de su primer hijo. No obstante, los resultados de la presente investigación no coinciden con los hallazgos de Cupa \& Riazuelo-Deschamps (2001) respecto a que el hijo sería considerado un rival edípico ante el afecto de la madre. Sin embargo, los padres del estudio sí consideran a la madre como una rival ante el afecto y la atención del hijo, lo cual no aparece reportado en las investigaciones revisadas $\mathrm{y}$ puede ser un aspecto relevante a profundizar en investigaciones venideras.

Respecto de los cambios en la identidad del padre con la llegada del primer hijo, los resultados arrojan dos posibilidades principales: la experiencia de entrega total por el hijo como una experiencia de entrega gratificante y de auto-trascendencia; o vivida como una pérdida de libertad asociada a sentimientos de tristeza e inadecuación. La paternidad como entrega total coincide con los estudios latinoamericanos de masculinidad (Fuller, 2000b), tanto desde el punto de vista de la presencia disponible para entregar afecto y cuidado al hijo, como de brindar las condiciones materiales necesarias para que el hijo se desarrolle. Los estudios señalan también que el hecho de ser padre implica el cierre de la etapa juvenil y el comienzo de la vida adulta (Fuller, 2000b). De acuerdo a la presente investigación, esto ocurre solo cuando hay efectos positivos en la identidad de los padres y adquieren una visión trascendente de la vida. Esta visión implica adquirir mayor madurez, más energía, aumentar la autoestima y sentirse más acompañado.

Ante estas dos posibilidades, surge la pregunta: ¿Qué factores inciden en que la experiencia de postergación de sí mismo sea vivida como auto-trascendencia o pérdida de libertad? El cariz negativo se daría en el caso de que el hijo no esté dentro de los planes del proyecto vital propio o exista una relación de pareja percibida como negativa. Escobar y Muñoz (1995) señalan que los hombres que logran incorporar a su hijo en su proyecto vital significan de manera positiva la paternidad. Sin embargo, a partir del análisis de los resultados de la presente investigación, se puede concluir que el hecho de incorporar o no al hijo en el proyecto vital estaría condicionado por el anhelo de ser padre. En otras palabras, la idea de ser padre estaría incorporada previamente como parte del proyecto vital en los padres que tienden a significar de buena forma la llegada de su primer hijo.

La importancia dada a la madre del hijo como influencia fundamental en el vínculo con él es concordante con hallazgos sobre representaciones sociales, tanto chilenos (Escobar \& Muñoz, 1995; Gallardo et al., 2006) como de otros países latinoamericanos (Alatorre \& Luna, 2000; Fuller, 2000a; Mora et al., 2005), los que señalan que el hijo es visto en el contexto de una relación de pareja-familia. Estos hallazgos coinciden, a su vez, con los de investigaciones de observación de interacciones precoces (Belsky et al., 1991; Volling \& Belsky, 1991). 
Respecto de las experiencias pasadas que influyen en el vínculo con el hijo, tanto el propio padre como la propia madre juegan un papel fundamental, ya sea por imitación o diferenciación, lo que coincide con los resultados de las investigaciones de Cupa y Riazuelo-Deschamps (2001), Fuller (2000a), Gallardo et al. (2006) y Olavarría (2000). Además, los padres de la presente investigación esperan superar a los propios padre y madre, estableciendo una mejor relación con su hijo de la que ellos tuvieron en su infancia, en especial respecto de ser más expresivos en el afecto y de estar presentes para a su hijo, lo que, a su vez, coincide con la representación social de la nueva paternidad planteada por Gallardo et al. (2006).

Desde una mirada metodológica, la principal limitación del estudio dice relación con la muestra, ya que solo se tuvo acceso a padres que presentaban interés en participar y se movilizaron para ello, siendo representativa del grupo de padres altamente involucrados afectivamente con su hijo y que disponían de tiempo para poder acudir a las entrevistas. Ello pudo haber influido en el modelo teórico emergente presentado.

La importancia del contacto sensorial directo con el hijo y, por lo tanto, de la presencia disponible que requiere el padre para dicho contacto y para facilitar una vinculación gratificante con su hijo, plantea una serie de interrogantes a ser resueltas, tanto por los propios padres en su vida personal como por los programas sociales que buscan promover la participación activa del padre en la crianza. Una de ellas es ¿cómo poder compatibilizar la función de proveer materialmente al hijo sin perder por ello la relación con él ni sentirse excluido de la familia? La sociedad ha tomado una serie de medidas para ayudar a las mujeres a compatibilizar el trabajo y la maternidad (e.g., licencias pre y postnatales, permisos laborales durante la lactancia). Sin embargo, estas medidas no están presentes en el caso de los padres. Otro desafío se presenta en los casos en que la relación con la madre es distante o conflictiva, debido a lo cual se obstaculiza el acceso del padre a su hijo durante el embarazo y los primeros meses de vida. Por lo tanto, ¿cómo un padre puede vincularse con su hijo, si no tiene acceso a interactuar con él a través del vientre materno durante el embarazo y, luego de nacido, puede verlo solo ocasionalmente? Tal vez en estos casos la intervención debe incluir la relación padre-madre.

Dadas estas circunstancias, es importante cuestionarse si el hecho de que los hombres tengan dificultades para asumir una paternidad inesperada con una pareja ocasional o con la que haya una relación percibida como negativa (Gallardo et al., 2006) se deba solo a los remanentes de machismo presentes aún en la sociedad actual o es necesario ampliar esta visión e incorporar el concepto de que en la estructura de la experiencia vincular de un padre con su hijo, si no hay contacto sensorial directo, probablemente será muy difícil que exista al menos en los primeros meses de vida un involucramiento más allá de pagar la pensión alimenticia exigida por la ley (Gallardo et al., 2006).

Otro factor importante de considerar en intervenciones que pretenden facilitar el vínculo padre-hijo es cómo el hombre que no tiene el anhelo previo de ser padre logra incorporar a su hijo como parte de su proyecto vital. Este aspecto es relevante debido a que en el presente estudio se sugiere que dicho anhelo influye decisivamente en cómo se significan los cambios en la identidad del padre con la llegada del hijo, lo que, a su vez, influye en que la relación con el hijo se torne gratificante o no.

Por último, futuras investigaciones podrían profundizar en el aspecto del contacto sensorial directo para la experiencia vincular, especialmente en grupos de padres que no vivan con sus hijos pequeños y/o que no tengan relación de pareja con la madre de su hijo.

\section{Referencias}

Aguayo, F. (2003). Paternidades, políticas y programas. Revisión de acciones y medidas para promover la participación del padre en el cuidado y la crianza de sus hijos e hijas (Documento de circulación interna). Santiago, Chile: Fondo de las Naciones Unidas para la Infancia.

Alatorre, J. \& Luna, R. (2000). Significados y prácticas de la paternidad en la Ciudad de México. En N. Fuller (Ed.), Paternidades en América Latina (pp. 241-275). Lima, Perú: Pontificia Universidad Católica del Perú, Fondo Editorial. 
Barclay L., Donovan J. \& Genovese A. (1996). Men's experiences during their partner's first pregnancy: A grounded theory analysis. Australian Journal of Advanced Nursing. 13(3), 12-23.

Barker, G. (2003). Men's participation as fathers in the Latin America and Caribbean region: $A$ critical literature review with policy consideration (Documento de circulación interna). Washington, DC: The World Bank.

Belsky, J., Yungblade, L., Robin, M. \& Volling, B. (1991). Pattern of marital change and parentchild interaction. Journal of Marriage and the Family, 53, 487-498.

Bowlby, J. (1998). El apego y la pérdida. Barcelona, España: Paidós.

Bradley, E., Mackenzie, M. \& Boath E. (2004). The experience of first-time fatherhood: A brief report. Journal of Reproductive and Infant Psychology, 22, 45-47. doi:10.1080/02646830310001643049

Clarke-Stewart, K. A. (1978). And daddy makes three: The father's impact on mother and young child. Child Development, 49, 466-478.

Cruzat, C. (2003). Significado de la paternidad en jóvenes varones del sector sur-oriente de Santiago (Tesis de Magíster no publicada), Escuela de Psicología, Pontificia Universidad Católica de Chile, Santiago, Chile.

Cruzat, C. \& Aracena, M. (2006). Significado de la paternidad en jóvenes varones del sector sur-oriente de Santiago. Psykhe, 15(1), 29-44. doi:10.4067/S0718-22282006000100003

Cupa, D. \& Riazuelo-Deschamps, H. (2001). La constellation paternelle: une étude pilote en période prénatale [La constelación paternal: un estudio piloto en el período prenatal]. Santé Mentale au Québec. Dossier Paternité et Santé Mentale, 26(1), 58-78.

Draper, J. (2002). 'It's the first scientific evidence': Men's experience of pregnancy confirmation. Journal of Advanced Nursing, 39, 563-570. doi:10.1046/j.1365-2648.2002.02325.x

Escobar, M. \& Muñoz, G. (1995). Estudio cualitativo de la experiencia de padres adolescentes (Tesis no publicada para optar al Título de Psicólogo), Escuela de Psicología, Pontificia Universidad Católica de Chile, Santiago, Chile.

Fachel, O. (2000). Impases de la paternidad: la reproducción desde la perspectiva masculina. En N. Fuller (Ed.), Paternidades en América Latina (pp. 309-331). Lima, Perú: Pontificia Universidad Católica del Perú, Fondo Editorial.

Federico, G. (2001). El embarazo musical. Buenos Aires, Argentina: Kier.

Federico, G. (2003). Música prenatal. Buenos Aires, Argentina: Kier.

Flick, U. (2004). Introducción a la investigación cualitativa. Madrid, España: Morata/Fundación Paideia Galiza.

Fuller, N. (2000a). Significados y prácticas de la paternidad entre varones urbanos del Perú. En N. Fuller (Ed.), Paternidades en América Latina (pp. 35-89). Lima, Perú: Pontificia Universidad Católica del Perú, Fondo Editorial.

Fuller, N. (2000b). Introducción. En N. Fuller (Ed.), Paternidades en América Latina (pp. 11-32). Lima, Perú: Pontificia Universidad Católica del Perú, Fondo Editorial.
Gallardo, G., Gómez, E., Muñoz, M. \& Suárez, N. (2006). Paternidad: representaciones sociales en jóvenes varones heterosexuales universitarios sin hijos. Psykhe, 15(2), 105-116. doi:10.4067/S071822282006000200010

Gibbons, C., Shiffman, R., Brophy-Herb, H., Fitzgerald, H. E., Omar, M. \& McKelvey, L. (2001). Une étude exploratorie. Interaction entre les dyades mère-nourrisson et père-nourrisson chez les couples à faible revenu [Un estudio exploratorio. La interacción entre las díadas madre-hijo y padre-hijo en las parejas de bajos ingresos]. Santé Mentale au Québec. Dossier Paternité et Santé Mentale, 26(1), 101-117.

Herzog, J. M. \& Lebovici, S. (1995). El padre. En S. Lebovici \& F. Weil-Halpern, La psicopatología del bebé (pp. 70-78). México, DF, México: Siglo XXI.

Kazura, K. (2000). Fathers' qualitative and quantitative involvement: An investigation of attachment, play, and social interactions. The Journal of Men's Studies, 9, 41-57. doi:10.3149/ jms.0901.41

Lamb, M. E. (1997). The development of father-infant relationships. En M. E. Lamb (Ed.), The role of the father in child development (pp. 104-120). Hoboken, NJ: John Wiley \& Sons.

Maldonado-Durán, J. M. (2005). El papel del padre durante la etapa perinatal y en la infancia temprana de los hijos. Topeka, KS: Kansas Association for Infant \& Early Childhood Mental Health. Extraído de http://www.kaimh.org/files/ monographs-articles/spanish/padre.htm

Marrone, M. (2001). La teoría del apego: un enfoque actual. Madrid, España: Psimática.

Mora, L., Otárola, C. \& Recagno-Puente, I. (2005). El hombre y la mujer frente al hijo: diferentes voces sobre su significado. Psykhe, 14(2), 119-132. doi: 10.4067/S0718-22282005000200010

Olavarría, J. (2000). Ser padre en Santiago de Chile. En N. Fuller (Ed.), Paternidades en América Latina (pp. 129-173). Lima, Perú: Pontificia Universidad Católica del Perú, Fondo Editorial.

Olavarría, J. (2001). Y todos querían ser (buenos) padres. Varones de Santiago de Chile en conflicto. Santiago, Chile: Facultad Latinoamericana de Ciencias Sociales.

Patton, M. Q. (1990). Qualitative evaluation and research methods. Thousand Oaks, CA: Sage.

Pedersen, D. (1992). El dilema de lo cuantitativo y lo cualitativo de las encuestas a los métodos rápidos de investigación en salud. En F. Lolas, R. Florenzano, G. Gyarmati \& C. Trejo (Eds.), Ciencias sociales y medicina. Perspectivas latinoamericanas (pp. 201-211). Santiago, Chile: Editorial Universitaria.

Pruett, K. D. (1998). Research perspectives: Role of the father. Pediatrics, 102, 1253-1261.

Stern, D. (1978). La primera relación madre-hijo. Madrid, España: Morata.

Stern, D. (1997). La constelación maternal: un enfoque unificado de la psicoterapia con padres e hijos. Barcelona, España: Paidós.

Stoleru, S. (1995). La parentificación y sus problemas. En S. Lebovici \& F. Weil-Halpern (Coord.), La psicopatología del bebé (pp. 92-111). México, DF, México: Siglo XXI. 
Strauss, A. \& Corbin, J. (2002). Bases de la investigación cualitativa: técnicas y procedimientos para desarrollar la teoría fundamentada. Medellín, Colombia: Universidad de Antioquia.

Viveros, M. (2000). Paternidades y masculinidades en el contexto colombiano contemporáneo, perspectivas teóricas y analíticas. En N. Fuller
(Ed.). Paternidades en América Latina (pp. 91127). Lima, Perú: Pontificia Universidad Católica del Perú, Fondo Editorial.

Volling, B. \& Belsky, J. (1991) Multiple determinants of father involvement during infancy in dualearner and single-earner families. Journal of Marriage and the Family, 53, 461-474.

Fecha de recepción: Enero de 2009.

Fecha de aceptación: Agosto de 2010. 